\title{
Improved Coded Cooperation Schemes for Wireless Communications
}

\author{
Yuanyuan Zhang, Yi Ma, and Rahim Tafazolli \\ Centre for Communication Systems Research \\ University of Surrey, UK, GU2 7XH. \\ Emails:\{yuanyuan.zhang, y.ma, r.tafazolli\}@surrey.ac.uk
}

\begin{abstract}
Cooperative communications can exploit distributed spatial diversity gain to improve link performance. When the message is coded at a low rate, source and relay can send different parts of a codeword to destination. This is referred to as the coded cooperation. In this paper, we propose two novel coded cooperation schemes for three-node relay networks, i.e., adaptive coded cooperation and ARQ-based coded cooperation. The former one needs the channel quality information available at source. The codeword splits adaptively to minimize the overall BER. The latter one is devised for relay network with erasure. In the first time slot, source sends a high-rate sub-codeword. Once destination reports the decoding errors, either source or relay can send one or two new bits selected from the mother codeword. Unlike random rateless erasure codes, such as Fountain code, the proposed scheme is based on the deterministic code generator and puncture pattern. It is experimentally shown that the proposed scheme can offer improved throughput in comparison with the conventional approach.
\end{abstract}

\section{INTRODUCTION}

Cooperative communications can exploit the distributed spatial diversity in multiuser systems [1]-[3]. This is particularly useful when each node can only be equipped with a single antenna. The cooperative strategies are often based on several classical relaying protocols such as amplify-and-forward (AF), decodeand-forward (DF), etc. Recently, Hunter and Nosratinia have reported a coded cooperation strategy [4]-[5]. The proposed approach is based on the DF relaying protocol. Unlike other DF-based cooperative strategies, source in coded cooperation does not send the full codeword in the first time slot. Instead, the full codeword (mother codeword) splits into two parts (sub-codewords), which can be sent by source and relay in a cooperative manner. This is analog to the hybrid ARQ technique, where either source or Node B can send one new sub-codeword once destination produces a request. It has been shown that coded cooperation outperforms other cooperative protocols in terms of the improved coding gain.

The conventional coded cooperation is proposed for scenarios without channel transmitter-side information. The puncture pattern for codeword splitting is fixed regardless of channel realization. It has been experimentally shown that the puncture pattern can affect the overall bit-error-rate (BER) performance [5]. The optimality depends on the channel realization. What is the optimum puncture pattern for a channel realization? How much performance gain the optimum design can offer? These questions motivate us to investigate the adaptive puncture pattern for coded cooperation (or called adaptive coded cooperation). Moreover, in many practical systems such hybrid ARQ, retransmission is required only when the receiver detects errors and makes a request. Either source or relay can send a new sub-codeword until there is no error at the receiver. This is a typical case for a communication channel with erasures. Random rateless erasure codes, such as Fountain codes, can offer the near network-capacity performance for these scenarios [6]. Here, we borrow the idea of Fountain codes to propose an ARQ-based coded cooperation strategy. Indeed, there is already some work combining Fountain codes with relay networks [7]. Our objective is to investigate what performance the deterministic puncture codes can offer for the erasure channels.

In detail, our contributions are mainly in two folds: 1) Assuming the channel quality information for three links are available at source, we propose the optimum puncture pattern to minimize the overall BER. It is found that the proposed adaptive coded cooperation can offer up to $2 \mathrm{~dB}$ performance gain in comparison with the conventional coded cooperation. 2) We propose an ARQ-based coded cooperation strategy, in which either source or relay sends 1 or 2 new coded bits for each request until the whole mother codeword is transmitted to destination. It is found that the proposed approach can 


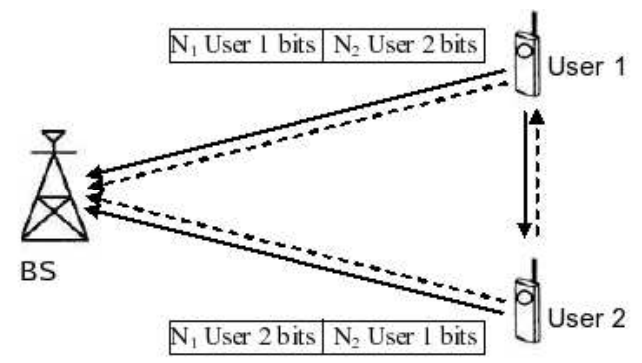

Fig. 1. System model for coded cooperation transmission

offer significantly improved throughput with reasonably good BER performance.

\section{System Model}

Here we consider the model of coded cooperation system as shown in Fig 1, with two mobile users, User1, User2 and base station (BS) in the system. Both users have data to be transmitted to BS. The channels among them are all modeled as independent slow Rayleigh fading channel.

Suppose there are a total of $K$ information bits per source block, and each block is encoded with an error correction code with rate $R$, and the total bits per source block is $N$. The two users cooperate by dividing the transmission of their $N$ bit codewords into two frames. In the first frame, each user transmits a rate $R_{1}>R$ codeword with $N_{1}=K / R_{1}$ bits. Each user receives and decodes the partner's first frame. If the user successfully decodes the partner's rate $R_{1}$ codeword (checked by CRC), the user computes and transmits $N_{2}$ additional parity bits for the partner's message in the second frame $\left(N_{1}+N_{2}=N\right)$. Whenever a user is unable to successfully decode its partner's message, the user will revert to a non-cooperative model by transmitting $N_{2}$ parity bits for his own message.

Performance results reported in [5] were obtained via a simple but effective implementation of coded cooperation using RCPC codes [8]. In this implementation, the overall rate $R$ code is a given RCPC code mother code. The codeword for the first $N_{1}$ bits is obtained by applying the puncturing matrix corresponding to rate $R_{1}$, and the additional parity $N_{2}$ bits transmitted in the second frame are those bits that are left over after puncturing in the first frame.

Because the transmission is symmetrical for the users, so in the rest of this paper, we take the transmission process for User1 only as an example. User1 serves as source (s), user2 as relay (r), and BS as destination (d) in a basic 3-node relaying system.

Although in [8], many possible puncture patterns are given for a certain mother code, but in [5], fair opportunity puncturing is mainly considered, that is $N_{1}=N_{2}=N / 2$. Some simulations are given with the puncture pattern satisfied $N_{1}=3 N_{2}=3 N / 4$ to show the different performance by different puncture patterns. However, how different puncture patterns perform in different channel realizations is not well investigated. In the following sections, we propose two improved schemes in two ways. The first is to change $N_{1}$ and $N_{2}\left(N_{1} / N_{2}\right.$ is defined as puncture ratio) under $N_{1}+N_{2}=N$, according to the instantaneous channel quality information (SNR) at source to minimize the overall BER. The other is to increase the overall code rate with $N_{1}+N_{2}<N$ on a ARQ basis, resulting in throughput improvement.

\section{ADAPTIVE CODED COOPERATION}

\section{A. Algorithm Description}

As provided by [8], one mother convolutional code can produce several RCPC codes by different puncturing. For example, the mother code is rate $R=1 / 4$ convolutional code with constraint length $M=4$. If the puncture period $P=8$, there are 13 different puncture patterns, with puncture matrix shown in Table I.

Assume the instantaneous SNR of all three channels is known to source. The algorithm can be described in the following several steps:

Step 1. Based on the instantaneous SNR, source calculates the overall system BERs for all puncture patterns.

Step 2. Source searches for the puncture pattern corresponding to the minimum BER performance to use for puncturing.

Step 3. Once the puncture pattern has been decided, thereafter, the transmission is processing in similar way with the conventional coded cooperation. An important difference is that when we choose the last puncture pattern in Table, we use only source to transmit the full codeword even if relay can decode the message from the source correctly. This will probably happens when rd link is bad enough, the proposed scheme can avoid possible severe performance degradation caused by poor rd link in conventional coded cooperation.

In Fig. 2, we show a frame division comparison between the conventional coded cooperation and our proposed schemes. For the conventional one, the whole frame, $\mathrm{N}$ bits, is divided into fixed $N_{1}$ and $N_{2}$ bits. The code rate for the whole codeword, 1 st and 2 nd frame are fixed to be $R, R_{1}$ and $R_{2}$. But for adaptive coded cooperation, there seems to be a slider, who decides the 
TABLE I

PUNCTURE TABLE FOR $r=1 / 4, M=4, P=8$

\begin{tabular}{|c|c|c|c|c|c|c|c|c|}
\hline code rate & punc & ure & nat & & & & & \\
\hline \multirow{4}{*}{$8 / 9$} & 1 & 1 & 1 & 1 & 0 & 1 & 1 & 1 \\
\hline & 1 & 0 & 0 & 0 & 1 & 0 & 0 & 0 \\
\hline & 0 & 0 & 0 & 0 & 0 & 0 & 0 & 0 \\
\hline & 0 & 0 & 0 & 0 & 0 & 0 & 0 & 0 \\
\hline \multirow{4}{*}{$8 / 10$} & 1 & 1 & 1 & 1 & 1 & 1 & 1 & 1 \\
\hline & 1 & 0 & 0 & 0 & 1 & 0 & 0 & 0 \\
\hline & 0 & 0 & 0 & 0 & 0 & 0 & 0 & 0 \\
\hline & 0 & 0 & 0 & 0 & 0 & 0 & 0 & 0 \\
\hline \multirow{4}{*}{$8 / 12$} & 1 & 1 & 1 & 1 & 1 & 1 & 1 & 1 \\
\hline & 1 & 0 & 1 & 0 & 1 & 0 & 1 & 0 \\
\hline & 0 & 0 & 0 & 0 & 0 & 0 & 0 & 0 \\
\hline & 0 & 0 & 0 & 0 & 0 & 0 & 0 & 0 \\
\hline \multirow{4}{*}{$8 / 14$} & 1 & 1 & 1 & 1 & 1 & 1 & 1 & 1 \\
\hline & 1 & 1 & 1 & 0 & 1 & 1 & 1 & 0 \\
\hline & 0 & 0 & 0 & 0 & 0 & 0 & 0 & 0 \\
\hline & 0 & 0 & 0 & 0 & 0 & 0 & 0 & 0 \\
\hline \multirow{4}{*}{$8 / 16$} & 1 & 1 & 1 & 1 & 1 & 1 & 1 & 1 \\
\hline & 1 & 1 & 1 & 1 & 1 & 1 & 1 & 1 \\
\hline & 0 & 0 & 0 & 0 & 0 & 0 & 0 & 0 \\
\hline & 0 & 0 & 0 & 0 & 0 & 0 & 0 & 0 \\
\hline \multirow{4}{*}{$8 / 18$} & 1 & 1 & 1 & 1 & 1 & 1 & 1 & 1 \\
\hline & 1 & 1 & 1 & 1 & 1 & 1 & 1 & 1 \\
\hline & 1 & 0 & 0 & 0 & 1 & 0 & 0 & 0 \\
\hline & 0 & 0 & 0 & 0 & 0 & 0 & 0 & 0 \\
\hline \multirow{4}{*}{$8 / 20$} & 1 & 1 & 1 & 1 & 1 & 1 & 1 & 1 \\
\hline & 1 & 1 & 1 & 1 & 1 & 1 & 1 & 1 \\
\hline & 1 & 1 & 0 & 0 & 1 & 1 & 0 & 0 \\
\hline & 0 & 0 & 0 & 0 & 0 & 0 & 0 & 0 \\
\hline \multirow{4}{*}{$8 / 22$} & 1 & 1 & 1 & 1 & 1 & 1 & 1 & 1 \\
\hline & 1 & 1 & 1 & 1 & 1 & 1 & 1 & 1 \\
\hline & 1 & 1 & 1 & 0 & 1 & 1 & 1 & 0 \\
\hline & 0 & 0 & 0 & 0 & 0 & 0 & 0 & 0 \\
\hline \multirow{4}{*}{$8 / 24$} & 1 & 1 & 1 & 1 & 1 & 1 & 1 & 1 \\
\hline & 1 & 1 & 1 & 1 & 1 & 1 & 1 & 1 \\
\hline & 1 & 1 & 1 & 1 & 1 & 1 & 1 & 1 \\
\hline & 0 & 0 & 0 & 0 & 0 & 0 & 0 & 0 \\
\hline \multirow{4}{*}{$8 / 26$} & 1 & 1 & 1 & 1 & 1 & 1 & 1 & 1 \\
\hline & 1 & 1 & 1 & 1 & 1 & 1 & 1 & 1 \\
\hline & 1 & 1 & 1 & 1 & 1 & 1 & 1 & 1 \\
\hline & 1 & 0 & 0 & 0 & 1 & 0 & 0 & 0 \\
\hline \multirow{4}{*}{$8 / 28$} & 1 & 1 & 1 & 1 & 1 & 1 & 1 & 1 \\
\hline & 1 & 1 & 1 & 1 & 1 & 1 & 1 & 1 \\
\hline & 1 & 1 & 1 & 1 & 1 & 1 & 1 & 1 \\
\hline & 1 & 0 & 1 & 0 & 1 & 0 & 1 & 0 \\
\hline \multirow{4}{*}{$8 / 30$} & 1 & 1 & 1 & 1 & 1 & 1 & 1 & 1 \\
\hline & 1 & 1 & 1 & 1 & 1 & 1 & 1 & 1 \\
\hline & 1 & 1 & 1 & 1 & 1 & 1 & 1 & 1 \\
\hline & 1 & 1 & 1 & 0 & 1 & 1 & 1 & 0 \\
\hline \multirow{4}{*}{$8 / 32$} & 1 & 1 & 1 & 1 & 1 & 1 & 1 & 1 \\
\hline & 1 & 1 & 1 & 1 & 1 & 1 & 1 & 1 \\
\hline & 1 & 1 & 1 & 1 & 1 & 1 & 1 & 1 \\
\hline & 1 & 1 & 1 & 1 & 1 & 1 & 1 & 1 \\
\hline
\end{tabular}

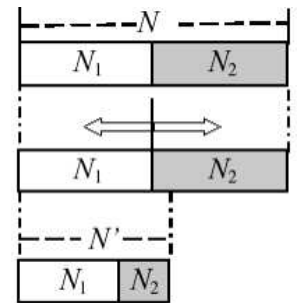

Original Coded Cooperation

Adaptive Coded Cooperation

Punctured Coded Cooperation

Fig. 2. Frame Structure Comparison

puncture ratio. In this case, the total codeword size and code rate are fixed to be $N$ and $R$ respectively. But $N_{1}$ and $N_{2}\left(R_{1}\right.$ and $\left.R_{2}\right)$ are various according to the various positions of the slider. If the slider slides to the left, it means relay will send a bigger portion of the codeword, and if the slider slides to the right, source will send a bigger portion. How to decide the position of slider (puncture ratio) is the most difficult thing of this scheme.

\section{B. BER prediction}

Like inverting the BER performance by the modulation size to get the boundary for choosing of different modulation schemes in adaptive modulation, inversion of overall BER should be done to get the boundary for different puncture ratio/pattern. But as for coded system, the BER performance of the system is too complicated to be easily invertible. Thus, we consider making BER prediction for all possible puncture patterns based on the instantaneous SNR, and then choose the puncture pattern with the minimum predict BER performance.

As convolutional code is used in the system, the best way to predict the BER is to use pairwise error probability.

The pairwise error probability of BPSK system can be written as [9],

$$
P(d \mid \gamma)=Q \sqrt{2 \sum_{n \in \varpi} \gamma(n)}
$$

where, $d$ is the Hamming distance between the transmitted codeword and the erroneously decoded codeword, and the set $\varpi$ is the set of all $n$ for which the received data is wrong.

Thus, in slow fading channels, as we consider here, SNR is constant over block, so, thus the above equation can be simplified as $Q(\sqrt{2 d \gamma})$.

The block error probability for a terminated convolutional code is bounded by [5],

$$
P_{\text {block }}(\gamma) \leq 1-\left(1-P_{E}(\gamma)\right)^{B} \leq B \cdot P_{E}(\gamma)
$$


where $B$ is the number of trellis branches in the codeword. In general, for a rate- $1 / \mathrm{n}$ convolutional code (or obtained by puncturing a rate- $1 / \mathrm{n}$ code), $B$ is equal to source block length $K . P_{E}(\gamma)$ is the error event probability that can be written as

$$
P_{E}(\gamma) \leq \min \left\{1, \sum_{d=d_{\min }}^{\infty} a_{d} P(d \mid \gamma)\right\}
$$

where $a_{d}$ is the number of error events with a Hamming distance $d$.

The conditional BER is bounded by

$$
P_{b}(\gamma) \leq \frac{1}{k_{c}} \sum_{d=d_{\min }}^{\infty} c_{d} P(d \mid \gamma)
$$

where, $c_{d}$ is the number of information bit errors for codewords with Hamming distance $\mathrm{d}$, and $k_{c}$ is the number of input bits for each branch of the code trellis.

The overall end-to-end performance of coded cooperation is

$P_{e}=P_{\text {block }}\left(\gamma_{s r}\right) P_{b}\left(\gamma_{s d}\right)+\left(1-P_{\text {block }}\left(\gamma_{s r}\right)\right) P_{b}\left(\gamma_{s d}, \gamma_{r d}\right)$

where $\gamma_{s r}, \gamma_{s d}$ and $\gamma_{r d}$ are the SNR of source-relay, source-destination and relay-destination link respectively, $P_{\text {block }}\left(\gamma_{s r}\right)$ is the block error rate of the sr link, $P_{b}\left(\gamma_{s d}\right)$ is the BER of the sd link if source sends the whole codeword without cooperation, and $P_{b}\left(\gamma_{s d}, \gamma_{r d}\right)$ is the BER at destination if source and relay transmit one portion each. $P_{b l o c k}\left(\gamma_{s r}\right)$, and $P_{b}\left(\gamma_{s d}\right)$ can be easily obtained using (3) and (4). As for $P_{b}\left(\gamma_{s d}, \gamma_{r d}\right)$, if $d_{1}$ and $d_{2}$ are the numbers of the error bits transmitted through source and relay user's channel respectively, such that $d_{1}+d_{2}=d . P_{b}\left(\gamma_{s d}, \gamma_{r d}\right)$ can be formulated as [10]

$P_{b}\left(\gamma_{s d}, \gamma_{r d}\right)=\frac{1}{k_{c}} \sum_{d=d_{\text {free }}} \frac{\left(\begin{array}{c}N_{1} \\ d_{1}\end{array}\right)\left(\begin{array}{c}N_{2} \\ d_{2}\end{array}\right)}{\left(\begin{array}{c}N \\ d\end{array}\right)} c_{d} P\left(d_{1}, d_{2} \mid \gamma_{s d}\right.$

Substituting (2)-(4) and (6) into (5), we can predict the BER for every puncture pattern. The puncture pattern with the smallest BER will be selected to finish the cooperative transmission.

\section{ARQ-BASED CODED COOPERATION}

Targeting to improve the throughput of the system, we propose another coded cooperation scheme. If destination can receive the data successfully using higher

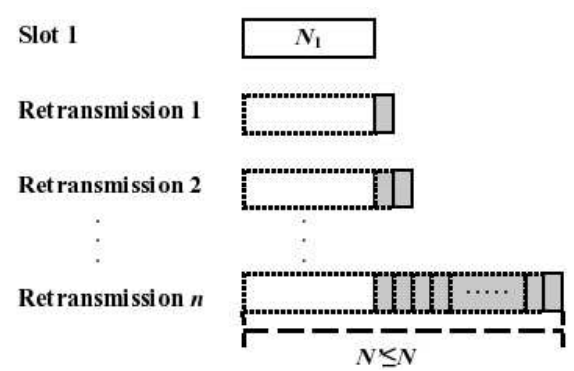

Fig. 3. Frame Structure Comparison

code rate $R^{\prime}>R$, high rate code with less redundan bits should be used to improve the throughput.

Looking through the puncture matrix for RCPC codes with code rates from high to low in Table I, the difference of matrix is shown in boldface. We can conclude that every bit in the high rate code appears in the low rate code; a lower rate codeword is formed just by selecting 1 or 2 more bits from the mother code compared with the adjacent higher rate code. Borrowing the idea of rartless code in relay system [7], our proposed scheme uses this observation of RCPC code to retransmit only extra 1 or 2 bits when required on an ARQ basis.

The transmission procedure is shown in the Fig 3, and only the data block with solid line is transmitted each time. In the 1 st slot, the highest rate code (i.e. rate- $8 / 9$ code in Table I) with length $N_{1}$ is sent to destination. If destination can't decode the message correctly, relay (if it can receive the data correctly) sends only extra bits to form the adjacent lower rate code (comparing rate$8 / 9$ and rate- $8 / 10$ code, only the very fifth bit of every whole codeword is transmitted to destination in the 2nd slot). If relay can't decode correctly, source does the retransmission and both relay and destination will try to decode after each receiving from source. Once relay get the data correctly, it will replace source to do the retransmission. This process continues until destination can decode successfully or it reaches the maximum retransmission time constraint. The total retransmission bits is summed to be $N_{2}$, and the resulting whole codeword is made up of $N_{1}+N_{2}=N^{\prime} \leqslant N$ bits, with code rate $R^{\prime} \geqslant R$. Obviously, the system throughput is improved averagely.

In this scheme, no instantaneous channel information is needed at source. As CRC is already exploited in coded cooperation system, only ARQ retransmission request is an extra need.

Fig.2 also shows the frame structure of this scheme. The whole transmission codeword size $N^{\prime}$ is variable (as 


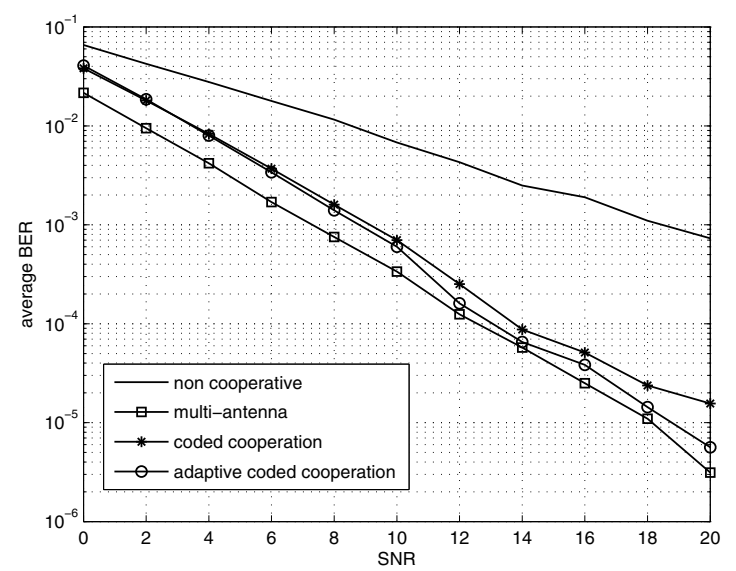

Fig. 4. BER Performance comparison for adaptive coded cooperation

well as rate $R$ ), and smaller or equals to $N$.

\section{NumericAl Results}

In this section, we present numerical results for the proposed two improved coded cooperation schemes in BPSK system over independent flat Rayleigh fading channels. We set the block size of information bits to $K=32$. We use the RCPC code and the puncture patterns as shown in Table I. Soft-decision Viterbi decoding algorithm is used as the decoding technique.

Adaptive coded cooperation scheme

In this simulation, we consider the three channels involved are with the same SNR, ranging from $0 \mathrm{~dB}$ to $20 \mathrm{~dB}$. For simplicity, we don't consider all possible puncture patterns in the simulation, we make our selection from the puncture code with rate 8/9, 8/16, 8/24 and non-cooperative system.

In the figures, 'multi-antenna' is a special case of coded cooperation with perfect sr channel. From Fig. 4, we can observe the BER improvement of the proposed adaptive coded cooperation over the conventional coded cooperation. Besides, we only consider 4 possible puncture patterns in the simulation, and the performance will be further improved if we consider all 13 puncture patterns.

In Fig. 5, we do the full selection from all 13 puncture patterns. The sd and rd channel have the same SNR from $0 \mathrm{~dB}$ to $20 \mathrm{~dB}$, and $\mathrm{sr}$ channel is fixed to be $20 \mathrm{~dB}$. The proposed scheme outperforms the conventional coded cooperation, and even better than the multiantenna system. This benefits from the knowledge of the instantaneous SNR at the transmitter, which can make

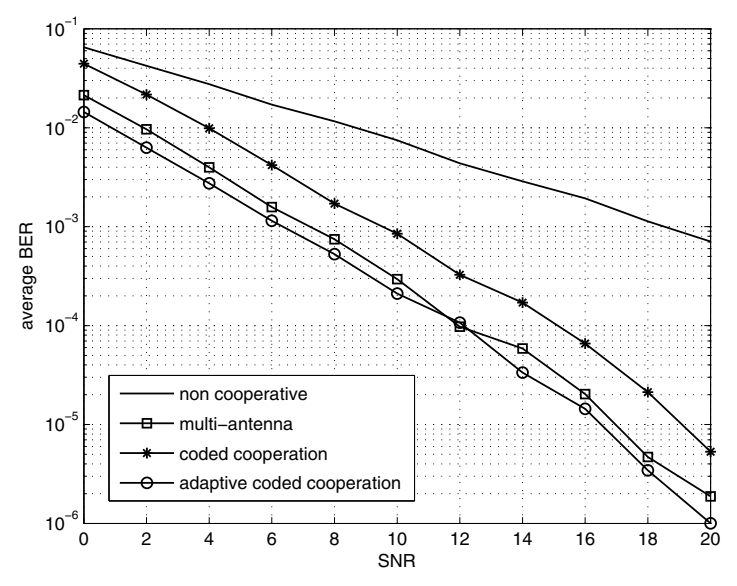

Fig. 5. BER Performance comparison for adaptive coded cooperation

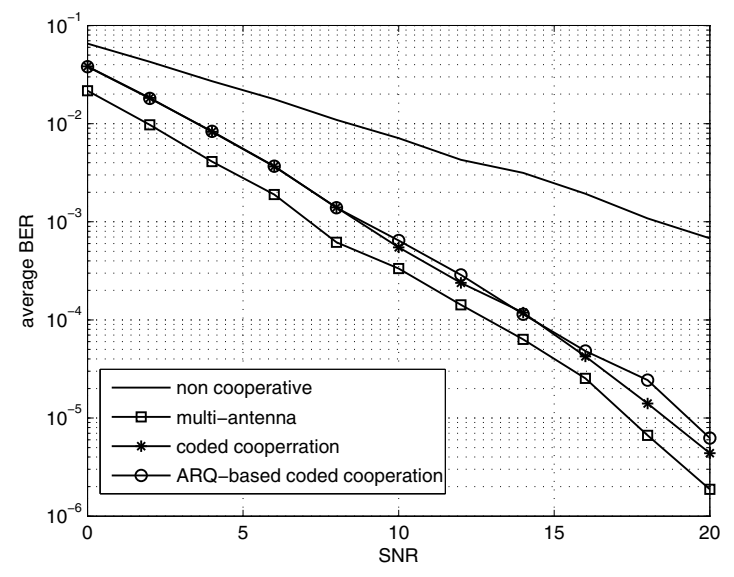

Fig. 6. BER Performance comparison for ARQ-based coded cooperation

the distribution of the codeword in a more optimal way compared with multi-antenna system.

\section{ARQ-based coded cooperation scheme}

In this simulation, the three channels are assumed to be with the same SNR, ranging from $0 \mathrm{~dB}$ to $20 \mathrm{~dB}$. To keep the lowest code rate for ARQ-based coded cooperation scheme the same with normal coded cooperation, we set the maximum retransmission time to be 12. This keeps fair comparison of our scheme with the conventional scheme. The simulation results are shown in Fig. 6 and Fig. 7.

Fig. 6 shows the BER performance of the schemes, and the ARQ-based coded cooperation can achieve similar BER performance with conventional coded cooperation. For conventional coded cooperation, the overall code rate is fixed to be 1/4, and Fig. 7 gives the average 


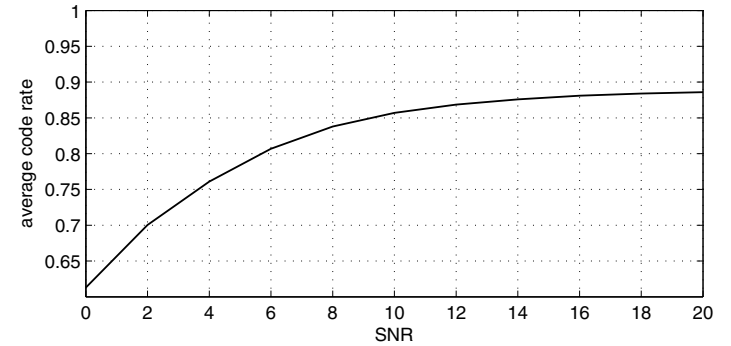

Fig. 7. Average coding rate for $\mathrm{AQR}$-based coded cooperation

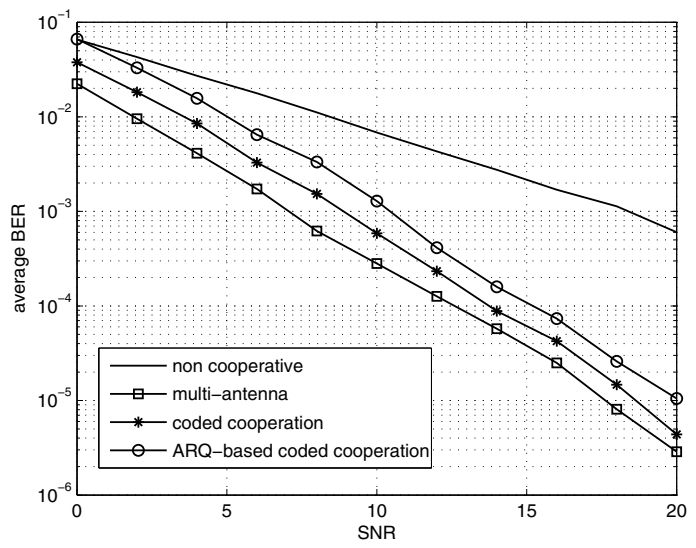

Fig. 8. BER Performance comparison for ARQ-based coded cooperation

code rate for the ARQ-based coded cooperation scheme, which is much higher than 1/4. So ARQ-based coded cooperation can provide a significant improvement in throughput while keeping the similar BER performance.

In practical system, retransmission for 12 times is not appropriate; but as shown in Fig. 7, from 10db to $20 \mathrm{~dB}$, the average code rate is larger than 0.85 , close to the highest code rate $8 / 9$, and this means it's quite rare for the transmitter to do the retransmission 12 times. If we set the maximum retransmission time to be smaller, we can get higher throughput gain with some scarification of the BER performance. Fig. 8 and Fig. 9 show the performance with the maximum retransmission time of 8. In this case, the ARQ-based coded cooperation is just about $1 \mathrm{~dB}$ less than coded cooperation at the BER performance. So for system with less BER performance requirement, we can set the retransmission time constraint to be smaller to reduce the complexity.

In these simulations, we use the RCPC code shown in Table I, but these two schemes work for other RCPC codes as well. We can choose other mother code and

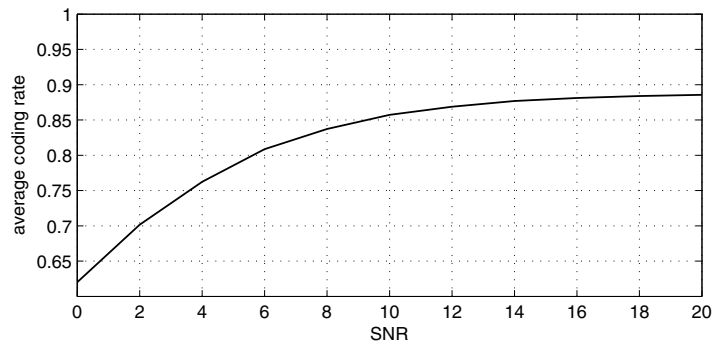

Fig. 9. Average coding rate for ARQ-based coded cooperation

search for good RCPC codes using the method introduced in [8].

\section{CONCLUSION}

In this paper, we have proposed two coded cooperation schemes - adaptive coded cooperation and ARQ-based coded cooperation, which can improve the BER and throughput performance respectively compared with the conventional coded cooperation system. The simulation results confirm our proposals by showing great improvement over the conventional scheme.

\section{REFERENCES}

[1] J. N. Laneman, D. N. C. Tse, and G. W. Wornell, "Cooperative diversity in wireless networks: Effi cient protocols and outage behaviour," IEEE Transactions on Information Theory, vol. 50, no. 12, pp. 3062-3080, Dec 2004.

[2] A. Sendonaris, E. Erkip, and B. Aazhang, "User cooperation diversity - part i: System description," IEEE Transcations on Communications, vol. 51, no. 11, pp. 1927-1938, Nov 2003.

[3] — , "User cooperation diversity - part ii: Implementation aspects and performance analysis," IEEE Transcations on Communications, vol. 51, no. 11, pp. 1939-1948, Nov 2003.

[4] A. Nosratinia, T. E. Hunter, and A. Hedayat, "Cooperative communication in wireless networks," IEEE Communications Magazine, vol. 42, no. 10, pp. 74-80, Oct 2004.

[5] T. E. Hunter and A. Nosratinia, "Diversity through coded cooperation," IEEE Transactions on Wireless Communications, vol. 5, no. 2, pp. 283-289, Feb 2006.

[6] D. MacKay, "Fountain codes," IEE Proceedings on communications, vol. 152, no. 6, pp. pp.1062-1068, Dec. 2005.

[7] J. Castura and Y. Mao, "Rateless coding for wireless relay channels," IEEE Transaction on wireless communications, vol. 6 , no. 5, pp. pp.1638-1642, May 2007.

[8] J. Hagenauer, "Rate-compatible punctured convolutional codes (rcpc codes) and their applications," IEEE Transactions on Communications, vol. 36, no. 4, pp. 389-400, Apr 1988.

[9] M. K. Simon and M. S. Alouini, Digital communication over fading channels : a unified approach to performance analysis, 1st ed. John Wiley \& Sons, 2000.

[10] S. A. Zummo, "Performance analysis for coded cooperation diversity in wireless networks," Wireless communications and mobile computing, vol. 7, no. 4, pp. 473-491, Jul 2006. 